\title{
Surgery and anaesthesia in the South African context: Looking forward
}

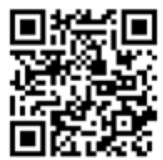

Historically considered an expensive, inefficient and limited public healthcare initiative, the place of surgery as the 'forgotten stepchild' of public health now leaves almost 5 billion individuals worldwide unable to access safe, affordable surgery when needed. In his keynote address at the National Forum on Surgery and Anaesthesia, held at the University of the Witwatersrand in December 2015, Deputy Minister of Health Dr Mathume Phaala stated that the ideal of a long and healthy life for all South Africans cannot be achieved without improved access to safe surgery and anaesthesia, a goal necessitating improvements in existing infrastructure, information systems, financial management and leadership. This marked the introduction of a groundbreaking event - the launch of a sustained Global Surgery movement in South Africa (SA) aimed at stimulating discussion on and conceptualising the future of surgery in SA. Discussion focused on needs assessment, a review of the essential package of surgical and anaesthetic care offered at regional, district and tertiary levels, monitoring of policy implementation, and a movement from the ideals of the Millennium Development Goals to sustainable goals.

The launch of the National Forum on Surgery and Anaesthesia coincided with the SA launch of the Lancet Commission on Global Surgery (LCoGS). The LCoGS was created 2 years ago with the purpose of generating large-scale sustainable improvement in critical, neglected areas of global health and to inform and drive policy change. It aims for surgery to be integrated into properly functioning healthcare systems by addressing current gross inequities in global delivery of safe and affordable surgery and anaesthesia. The vision of the LCoGS is simple: universal access to safe and affordable surgical and anaesthetic care when needed. This goal is a moral and economic priority in the developing world. It is estimated that failure to provide safe, accessible and effective surgical and anaesthetic services may cost low- and middle-income countries (LMICs) up to USD12.3 trillion in lost productivity, translating into $2 \%$ of gross domestic product growth annually between 2015 and 2030, if left unaddressed.

Research by the LCoGS identifies six key areas that affect access to safe, effective and affordable surgery in LMICs. Access to timely surgery, specialist workforce density, surgical volume, the perioperative mortality rate, impoverishing expenditure and catastrophic expenditure represent significant barriers to surgical care. For example, specialist workforce density is a measure of the number of surgeons, anaesthetists and obstetricians (SAOs) per 100000 population. It is estimated that approximately $72 \%$ of the world's population live in countries with an SAO density of $<40 / 100000$. Data analysis by the LCoGS reveals that approximately 2 million specialist healthcare providers are needed to reach the goal of an SAO density of 40/100 000 in LMICs by 2030 . Using obstetrics as an example, the workforce indicator found that for each ten-unit increase in the density of SAOs, there is a $13.1 \%$ decrease in maternal mortality rate. In the SA context, it is clear that expansion of a skilled workforce capable of meeting population health needs is necessary, but will not resolve existing challenges. Human resource constraints are a well-recognised shackle preventing delivery of safe and effective healthcare. Responding to the shortage of healthcare professionals in SA, numerous training centres have undertaken the training of clinical associates. Although still a relatively new component of the healthcare workforce, these individuals have the potential to contribute significantly to the delivery of safe, timely surgery and anaesthesia in our context. In conjunction with an ongoing review of the current syllabus for undergraduate medical training geared towards the training of efficient generalists, these initiatives are examples of ways in which the SA medical community is already responding to public healthcare needs.

As noted by representatives of the Department of Health, the concept of surgery in SA needs to change in order for the targets of the LCoGS to be met. This acknowledgement calls for support for the concept of the acute care surgeon, capable of providing specialist-level general surgical care, as well as support for the training of more generalists, possibly in the form of career medical officers, and reversing the growing trend towards narrow subspecialist training seen among newer graduates. Further, the devolution of care may necessitate the use of cross-trained and skilled medical officers to undertake to provide a broad range of surgical and anaesthetic services at regional and even primary care levels. Numerous experts at the forum raised significant concerns regarding the decentralisation of care to primary and regional centres. Maternal and anesthetic mortality rates that vary widely between provinces and between regional and tertiary centres in SA were used to argue against the decentralisation of care. Notwithstanding the merits of arguments for and against particular interventions, the LCoGS and the National Forum on Surgery and Anaesthesia provide the first objective framework with which to analyse existing policy and propose and implement change.

Considering the challenges identified, developing a national road map for surgery and anaesthesia is a colossal task. Objective assessment of population needs is vital to developing any strategies aimed at addressing deficiencies in the current profile of service delivery. This assessment demands accurate and regular data collection and reporting. In spite of the existing demands on service delivery providers, an ongoing assessment of needs and the effectiveness of policy interventions provides an opportunity for innovation and to develop the relationship between tertiary, regional, peripheral and primary levels of care. As noted by the successful completion of the South African Surgical Outcomes Study, research of this nature is possible, even in our particular context.

In 2015, the World Health Organization acknowledged the critical place of surgery and anaesthesia in universal healthcare through its resolution on Strengthening Emergency and Essential Surgical Care and Anaesthesia as a Component of Universal Health Coverage. This resolution guides governments that are signatories to the resolution (including SA) to actively support and engage in improving surgical care. As noted by representatives of the National Treasury, surgical care providers need greater input at policy-making level in order to develop a financial model capable of meeting our population's surgical needs. To date, such engagement has not been realised.

The National Forum on Global Surgery and Anaesthesia presents the first step towards the objective acknowledgement of the challenges of providing safe, accessible, effective and affordable surgical and anaesthetic care in the SA context. Incorporating 
the input of local and international, surgical, anaesthetic and public health, academic and non-academic, and private and public stakeholders, the foundation to systematically discuss and tackle issues of infrastructure, workforce, service delivery, financing and information management challenges has been formed. It is now incumbent on us as the medical community to continue this momentum and effect real change.

\section{N Patel}

Department of Paediatric Surgery, Faculty of Health Sciences, University of the Witwatersrand, Johannesburg, South Africa

\section{Peffer}

Department of General Surgery, School of Medicine, Faculty of Health Sciences, University of Pretoria, South Africa

\section{A Leusink}

Chris Hani Baragwanath Academic Hospital, Johannesburg, South Africa

\section{N Singh}

Department of General Surgery, Faculty of Health Sciences, Sefakgo Makgathu University, Pretoria, South Africa

\section{Smith}

Department of General Surgery, Faculty of Health Sciences, University of the Witwatersrand, Johannesburg, South Africa

Corresponding author: N Patel (niravpatel44@gmail.com)

S Afr Med J 2016;106(2):135-136. DOI:10.7196/SAMJ.2016.v106i2.10529 